\title{
DOCTOR'S ROLE IN EARLY DETECTION OF DIABETIC RETINOPATHY AND PREVENTION OF BLINDNESS FROM ITS COMPLICATIONS
}

\author{
Subedi $\mathrm{S}^{*}$, Subedi K U*, Badhu B P* \\ * Rana-Ambika Shah Eye Hospital, Bhairahawa, ** BPKIHS, Dharan
}

\section{ABSTRACT}

Diabetic retinopathy (DR) is a microangiopathy, which is caused by chronic hyperglycemia, affecting the retinal arterioles, capillaries and venules, complications of which lead to incurable blindness.

Approximately $10 \%$ of the diabetic population has type I diabetes mellitus (DM) which is diagnosed before the age of 30 years $^{1}$ and rest is type II which is diagnosed after the age of 30 years. In UK $2 \%$ general population is affected by DM. In developed countries, diabetic retinopathy is an important and leading cause of blindness in working age group where as in developing western countries this figure occupy $12 \%$ of the blindness.

In developing countries like Nepal, cataract still remains a main cause of blindness and diabetes is not considered as a major problem. However due to a rapid urbanization and modernization of population, diabetes mellitus is becoming an endemic disease and bringing a new challenge in blindness reduction program.

Key Words: Early detection of DR, Classification of DR, Complications, Prevention of blindness.

\section{INTRODUCTION}

The exact cause of diabetic microangiopathy is unknown. It is believed that exposure to hyperglycemia over an extended period leads to several hematological and biochemical changes which ultimately cause loss of pericytes, basement membrane thickening and microvascular (endothelial) decompensation.

Hematological and biochemical abnormalities responsible for severity ${ }^{1-3}$ of retinopathy include:

- Increased platelet adhesiveness

- Increased erythrocytes aggregation

- Abnormal serum lipids

Address for correspondence :

Dr. Sudesh Subedi

Rana-Ambika Shah Eye Hospital, Siddharthanagar, Bhairahawa, Nepal

Email: ssubedi63@rediffmail.com

Received Date : 22 nd August, 2003

Accepted Date : $19^{\text {th }}$ May, 2005

- Defective fibrinolysis

- Abnormalities in serum and whole blood viscosities etc.

The diabetic retinopathy has features of microvascular occlusion leading to retinal ischemia and vascular leakage. The ischemic retina is responsible for abnormal new vessels formation in the retina and in optic disc, which later on may be main cause of marked loss of vision (vision $<5 / 60$ ).

Such conditions are vitreous hemorrhage, tractional macular detachment or combined mechanisms retinal detachments. 
Vascular leakage on the other hand is responsible for exudative maculopathy, cystoid macular oedema or clinically significant macular oedema, which causes mild to moderate loss of vision.

After about 20 years of diabetes, nearly all patient with type I diabetes mellitus and more than $60 \%$ of patient with type II diabetes mellitus have some degree of retinopathy. ${ }^{4}$

\section{CLASSIFICATION}

DR is classified on the basis of its severity. It has been attempted by Hirschberg in 1980 with the objective of recording the natural history of the disease, the time for intervention, and the mode of intervention and evaluation of its efficacy.

There are various classifications and most commonly used classifications ${ }^{3,5,6}$ in current practice used:

1. Air lie House classification

2. Kanski's classification - It includes following stages
a. Simple background DR (BDR)
b. Preproliferative DR (PPDR)
c. Proliferative DR (PDR)

3. ETDRS (early treatment DR study) -

This classification is made on the basis of Airlie House classification and it is the modern well accepted classification in the world. It includes:

a. Non proliferative (NPDR) - It has 4 types:

- $\quad$ Mild NPDR

- Moderate NPDR

- $\quad$ severe NPDR

- $\quad$ very severe NPDR

Findings of mild, moderate and severe NPDR are similar to BDR and findings of very sever NPDR similar to PPDR

b. Proliferative DR (PDR) -It has 2 types:

- early PDR

- $\quad$ high risk PDR
Fig. 1 : Mild NPDR with CSME Left Eye (Small arrow - Hg, Long arrow - CSME with hard exudate)
Fig. 2 : Moderate NPDR Left Eye

(Small arrow - Hg,

Long arrow - cotton wool spot)

Fig. 3 : Sever NPDR

(Double arrow - Venus Beading)

Fig. 4 : Very severe NPDR

(Double arrow - Venus Beading) 
Fig. 5 (a) : Early PDR

(All arrows - NVE)

Fig. 5 (b) : Early PDR

(All arrows - NVE)

\section{Fig. 6 : HR - PDR}

(All small arrows - NVE

Long arrow - SHH)
Fig. 7 : Normal Fundus of Right Eye

(No DR changes even after 19 yrs duration of Diabetes mellitus)

CLINICAL FEATURES AND EARLY DETECTION OF DR

The prevalence of retinopathy in diabetic population increases with the duration as well as with the type of diabetes and age of the patient.

It is rare to find DR in children of less than 10 years of age. ${ }^{7}$

Patient with DR changes may or may not have ocular symptoms. Mostly symptoms reflect the advanced stage of DR. However asymptomatic case is not excluded from the severity. It may have any stage of DR from no retinopathy to NPDR and HRPDR. Presentations of DR and fundus evaluation are as follows:

I. Asymptomatic-until macula is involved or VH or TRD occurs

II. Defective vision - mild to moderate visual loss usually is caused by macular involvement where as marked loss of vision is due to $\mathrm{VH}$ or macular TRD

III. Floaters - In diabetic patient having floaters of recent onset, VH should be suspected

IV. Fundus examination - There are several methods of fundus evaluation like direct ophthalmoscope, indirect ophthalmoscope, slit lamp biomicroscopy and fundus photography etc. Direct ophthalmoscopy is simple and better method to detect diabetic retinopathiy changes. In first check up it should be performed after pupillary dilation so that posterior pole is well visualized. For dilation, eye drops like $1 \%$ tropicamide or $2.5 \%$ to $10 \%$ phenylephrine can be used. In diabetic patient, posterior pole examination is very important where retinopathy signs have to be looked for, particularly in and around the optic disc, macular area, superior and inferior arcades and temporal to the macula. DR signs include micro aneurisms (early sign of DR), ${ }^{8}$ dot and blot hemorrhages, hard exudates, cotton wool spots, new vessels in and outsides the optic disc, vitreous or subhyaloid hemorrhages etc. (Table I). , $^{3,9,10}$ 
Table I : Severity of DR and its Fundus Features

\begin{tabular}{|c|c|}
\hline SEVERITY OF DR & FEATURES OF DR \\
\hline Mild NPDR & At least one micro aneurysm (MA), few retinal hemorrhage or hard exudates \\
\hline Moderate NPDR & $\begin{array}{l}\text { Features of mild NPDR with soft exudates (cotton wool spots), venus beeding (VB) and } \\
\text { intraretinal microvascular abnormalities (IRMA). }\end{array}$ \\
\hline Severe NPDR & $\begin{array}{l}\text { Features of moderate NPDR with one of the following: a. Hemorrhage / MA in all } 4 \\
\text { quadrants b. VB in } 2 \text { or more quadrants c. IRMA in at least in one quadrant. }\end{array}$ \\
\hline Very Severe NPDR & Any two or more of severe NPDR and absence of new vessels \\
\hline Early PDR & Presence of new vessels and criteria not met for HR - PDR \\
\hline High risk PDR (HR-PDR) & $\begin{array}{l}\text { neovascularization of optic disc (NVD) } \geq 1 / 3 \text { rd to } 1 / 2 \text { disc, or NVD with vitreous } \\
\text { Hemorrhage }(\mathrm{VH}) \text { and NVE } \geq 1 / 2 \text { disc area with pre-retinal hemorrhage or vitreous } \\
\text { hemorrhage. }\end{array}$ \\
\hline $\begin{array}{l}\text { Clinically significant } \\
\text { macular oedema (CSME) }\end{array}$ & $\begin{array}{l}\text { a. Retinal chickening located } \leq 500 \mu \text { from the center of the macula or } \\
\text { b. hard exudates with retinal thickening of the adjacent retina located } \leq 500 \mu \text { from the } \\
\text { center of macula or } \\
\text { c. a zone of retinal thickening } 1 \text { disc area or larger in size located } \leq \text { disc diameter from } \\
\text { the center of macula }\end{array}$ \\
\hline
\end{tabular}

If fundus evaluation is difficult due to cataract or any other reason, even after pupil dilation, it is advisable to be evaluated by an ophthalmologist.

\section{GUIDELINE FOR MANAGEMENT}

Although a lot of researches ${ }^{11}$ have been done, there is no single modality to prevent or cure DR and it is still under research. The ways to prevent moderate to severe visual loss in diabetes are:

1. Careful eye examination and regular follow-up

2. Referral to ophthalmologist or vitro-retinal surgeon for laser photocoagulation treatment or vitrectomy surgery

\section{EYE EXAMINATION AND FOLLOW UP SCHEDULE}

Once diabetes mellitus is diagnosed, physician should evaluate the fundus as described above or should refer to an ophthalmologist expert in retinal diseases or to the vitreoretinal surgeon for base line evaluation. In subsequent visits apart from regular fundus evaluation, patient's physician (family physician. general physician, pediatrician etc) can play a key role in prevention of rapid progression of DR by regular assessment of diabetes mellitus and other high risks factors ${ }^{12}$ e.g. diabetic nephropathy, hypertension, systemic infection, pregnancy, hyper-triglyceridemia or hyper-cholesterolemia, renal failure, renal transplantation etc. These uncontrolled conditions may lead to irreversible sight loss and even death of patient.

Hence it is important for patient's doctors* to plan a careful program of evaluation, education and follow-up of any ocular condition whether the patient is symptomatic or not in spite of having good vision. ${ }^{13}$

Eye examination and follow up schedules are described in Tables II and III. ${ }^{14,15}$

\section{REFERRAL TO THE OPHTHALMOLOGIST OR VITREORETINAL SURGEON}

Timely detection and identification of DR stage are crucial in its management. ${ }^{16}$ This ability entirely depends upon the experience and skill of the doctors (physician, ophthalmologist, and pediatrician) and also affected by the presence of hazy media (due to cataract), choroidal sclerosis and featureless retina. In such situations doctors can refer the case to a vitreoretinal surgeon.

There are few general criteria defined by ETDRS for referral and management of the diabetic retinopathy cases:17,18,19

a) Cases that need only observation and follow up (see table I, II and III). It includes following cases: mild, moderate and severe stages of DR.

b) Cases that need immediate laser treatment. It includes HRPDR, CSME and iris neovascularization.

c) Cases that need close observation or laser treatment. It includes very severe NPDR (preproliferative NPDR) and early PDR. If close follow-up is not possible or if it is associated with high risk conditions then laser treatment is advised. High risks condition are those factors which

Doctors* - It denotes those doctors who are involving in diabetic patient's treatment e.g. general physician, family physician, pediatrician, diabetologist, nephrologists, ophthalmologist etc. 
Table II : Eye Exam Schedule

Table III : Recommended Follow up Schedule in DR

progresses the diabetic retinopathy. e.g. Diabetic nephropathy, kidney transplantation, pregnancy, hypertension, cataract surgeries, long duration of DR etc.

d) Cases that need vitrectomy surgeries, e.g. nonresolving vitreous hemorrhage, early macular TRD etc.

Combined efforts of all doctors and strict adherence to above guideline and criteria can only make it possible to prevent unnecessary visual loss of patient with significant retinopathy. It will also be cost effective to the society and help to maintain normal life style of diabetic patients.

\section{ACKNOWLEDGEMENT}

Authors would like to thank Mr. Chitra K. Karna, Mr. Ganesh Thapa and Mr. Binod Pandey (Shree Rana - Ambika Shah Eye Hospital) for their generous help in computer work.

\section{REFERENCES}

1. The foundation of American academy of Ophthalmology. Basic and clinical science course, Retina and Vitreous. Section 12, 2000-2001, 88-110.

2. Jack J Kanski. Textbook of clinical ophthalmology. Fourth edition, 1999; 465 - 476.

3. Stephan J Ryan. Etiologic mechanism in diabetic retinopathy. Retina vol. II; Third edition; 1259-1349.

4. Klein R, Klein BE, most SE, et al. The Wisconsin Epidemiologic study of Daibetic Retinopathy: XVII. The 14 years incidence and progression of diabetic retinopathy and associated risk factors in type I diabetes. Ophthalmology, 1998, 105: 1801-1815.

5. Albert and Jacobiec. Principle and practice of Ophthalmology. Text book; second edition; page 1901-1907.

6. Steven M. Bloom, AJ Brucker. Laser surgery of the posterior segment. Lippincott-Raven publisher 1997; 39-68.
7. Jochmann C, Hammes HP. Epidemiology, pathogenesis and therapy of DR and maculopathy. Arztl, Forbid Qualitatssich. 2002 March, 96 (3): 167-74.

8. Friedenwald, JS: Diabeticretinopathy. Am. J. Ophthalmol 1950, 33: 11871199.

9. Early Treatment Diabetic Retinopathy Study Research Group: Photocoagulation for diabetic macular edema, ETDRS Reports No. 1, Arch Ophthalmol clin 1987, 27: 265-72.

10. Diabetic Retinopathy Study Research Group: Clinical application of diabetic retinopathy study (DRS), findings, DRS Report No. 8. Ophthalmology 1981, 88: 583-600.

11. Cunha-Vaz J. Lowering the risk of visual impairment and blindness. Diabet Med. 1988, 15 Suppl 4: 547-50.

12. Subedi S.. Cataract surgery and progression of diabetic retinopathy. JNMA, 2002; Vol.41: 433-437.

13. Massin P. Ocular complications in diabetes: towards standardizing screening and care. Rev. prat. 2001 Oct 15, 51(16): 1776-82.

14. Javitt Jc. Canner JK, et al. Cost effectiveness of current approaches to the control of retinopathy in type I diabetic. Ophthalmology 1989, 96: $255-64$.

15. Clein R. Eye care delivery for people with diabetes. Diabetes care (Commentary) 1994, 17: 614-615.

16. Lightman S, Towler Hm. Diabetic retinopathy. Clin cornerstone 2003, 5(2): 12-21.

17. Early Treatment Diabetic Retinopathy Study Research Group: ETDRS Design and baseline patient characteristics, ETDRS Report No. 7. Ophthalmology 1991, 98: 741-756.

18. ETDRS Research Group: Techniques for scatter and local photocoagulation treatment of DR, ETDRS Report No.3. Int Ophthalmol clin 1987, 27: 254-264.

19. Aiello LM. Perspectives on diabetic retinopathy. Am. J. Ophthalmol. 2003 Jul, 136 (1): 122-135. 